\title{
含 $\mathrm{MoFe}_{3} \mathrm{Se}_{4}$ 类立方烷核芯簇合物的合成 与结构表征
}

\section{张千锋 曹 荣 洪茂椿 ${ }^{* *}$ 刘汉钦 卢嘉锡}

(中国科学院福建物质结构研究所, 结构化学国家重点实验室, 福州 350002)

\begin{abstract}
摘要 室温下 $\left[\mathrm{Et}_{4} \mathrm{~N}\right]_{2} \mathrm{MoSe}_{4}, \mathrm{FeCl}_{2}$ 和 $\mathrm{R}_{2} \mathrm{NCS}_{2} \mathrm{Na}$ 在 $\mathrm{DMF}$ 和 $\mathrm{CH}_{3} \mathrm{CN}$ 混合溶剂中反应, 得 到含 $\mathrm{MoFe}_{3} \mathrm{Se}_{4}$ 核芯的 $\mathrm{Mo}-\mathrm{Fe}-\mathrm{Se}$ 簇合物 $\mathrm{MoFe}_{3} \mathrm{Se}_{4}\left(\mu-\mathrm{R}_{2} \mathrm{NCS}_{2}\right)_{2}\left(\mathrm{R}_{2} \mathrm{NCS}_{2}\right)_{4}\left(\mathrm{R}_{2}=\mathrm{Me}_{2}(\mathbf{1})\right.$, $\mathrm{Et}_{2}(2), \mathrm{C}_{4} \mathrm{H}_{8}(3)$ ). 化合物 2 的单晶 $\mathrm{X}$ 射线衍射分析表明, 其分子结构为 2 个桥式和 4 个螯合 $\mathrm{Et}_{2} \mathrm{NCS}_{2}{ }^{-}$配体包围的一个扭曲类立方烷 $\mathrm{M}_{4} \mathrm{Se}_{4}$ 簇核. 对 3 个化合物的 $\mathrm{CV}$ 进行 了表征, 它们在 DMSO 溶液中的电化学行为表现出了多电子可逆的传递过程.
\end{abstract}

\section{关键词 Mo-Fe-Se 簇合物 类立方烷 晶体结构 CV}

过去的 20 多年中, 对固氮酶催化中心模型的研究促进了结构不同的钼、铁、硫簇合物的 合成 ${ }^{[1]}$, 如单立方烷和双立方烷簇, 其中单立方烷簇合物是固氮酶活性中心很好的化学谱学模型 ${ }^{[2]}$. 近来的结构测定揭示固氮酶的每个活性中心包含双桥簇合物: 即 $4 \mathrm{Fe}: 4 \mathrm{~S}$ 和 $1 \mathrm{Mo}: 3 \mathrm{Fe}: 3 \mathrm{~S}$ 簇 为 $\mathrm{Fe}-\mathrm{Mo}$ 辅基及双 $4 \mathrm{Fe}: 4 \mathrm{~S}$ 立方烷为 $\mathrm{p}$-cluster ${ }^{[3]}$, 这些结果使得 $\mathrm{Fe}-\mathrm{S}$ 和 $\mathrm{Mo}-\mathrm{Fe}-\mathrm{S}$ 原子簇化学 的研究越来越引起人们的兴趣 ${ }^{[4]}$. 硒是自然界重要的痕量元素之一, 并且人们还发现它是许多 酶中的一种重要成分 ${ }^{[5]}$, 比如在细菌脱辅基蛋白、肾上腺和植物铁氧化还原蛋白中都发现含 有 $\mathrm{Fe}-\mathrm{Se}$ 簇的蛋白, 显示在自然界中可能存在 $\mathrm{Fe}-\mathrm{S}$ 或 $\mathrm{Mo}-\mathrm{Fe}-\mathrm{S}$ 蛋白的硒同类物 ${ }^{[6]}$. 到目前为 止, 用 Se 原子部分或全部取代 Fe-S 簇合物中 $\mathrm{S}$ 原子的研究已有许多报道, 而用硒取代 Mo$\mathrm{Fe}-\mathrm{S}$ 簇合物中的研究仅在几年前刚开始, 并只得到了少数的几个化合物, 如 Stiefel 和他的合

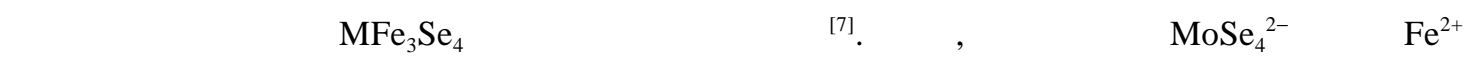
的反应, 并成功地合成出一系列单立方烷 Mo- $\mathrm{Fe}-\mathrm{Se}$ 簇合物.

本文报道系列单立方烷 $\mathrm{Mo}-\mathrm{Fe}-\mathrm{Se}$ 簇合物 $\mathrm{MoFe}_{3} \mathrm{Se}_{4}\left(\mu-\mathrm{R}_{2} \mathrm{NCS}_{2}\right)_{2}\left(\mathrm{R}_{2} \mathrm{NCS}_{2}\right)_{4}\left(\mathrm{R}_{2}=\mathrm{Me}_{2}(\mathbf{1})\right.$, $\operatorname{Et}(2), \mathrm{C}_{4} \mathrm{H}_{8}(3), \mathrm{R}_{2} \mathrm{NCS}_{2}{ }^{-}$称为 $\left.\mathrm{R}_{2} \mathrm{dtc}\right)$ 的合成与表征.

\section{1 实验}

\section{1 合成}

所有操作均在氮气保护下采用标准 Schlenk 技术进行, 市售 AR 级溶剂在使用前经纯化 并干燥处理. $\left[\mathrm{Et}_{4} \mathrm{~N}\right]_{2}\left[\mathrm{MoSe}_{4}\right]$ 按文献[8]加以改进, 并按我们前期报道的方法 ${ }^{[9]}$ 合成.

(1) $\left[\mathrm{MoFe}_{3} \mathrm{Se}_{4}\left(\mathrm{Me}_{2} \mathrm{dtc}\right)_{6}\right]$ (1)的制备. $\left[\mathrm{Et}_{4} \mathrm{~N}\right]_{2}\left[\mathrm{MoSe}_{4}\right](0.17 \mathrm{~g}, 0.25 \mathrm{mmol}), \mathrm{FeCl}_{2}(0.10 \mathrm{~g}, 0.79$ 
$\mathrm{mmol})$ 和 $\mathrm{Me}_{2} \mathrm{dtcNa}(0.23 \mathrm{~g}, 1.55 \mathrm{mmol})$ 溶于 $20 \mathrm{~mL} \mathrm{DMF}$ 中, 室温下搅拌 $8 \mathrm{~h}$ 后过滤, 滤液中 加入 $15 \mathrm{~mL} \mathrm{CH} 3 \mathrm{CN}$ 溶液; 再滤去形成的黑色沉淀物和无机盐. 放在冰箱于 $4^{\circ} \mathrm{C}$ 条件下, 2 周后 得到黑色棱柱状晶体, 用无水乙醇洗涤并真空干燥, 得 $0.15 \mathrm{~g}$ (产率为 $33.4 \%$ ). 按 $\mathrm{C}_{18} \mathrm{H}_{36} \mathrm{~N}_{6} \mathrm{MoFe}_{3} \mathrm{Se}_{4} \mathrm{~S}_{12}$ 计算值(\%): C, 16.6; H, 2.79; N, 6.46; Fe, 12.9; Mo, 7.38. 实验值(\% ): $\mathrm{C}$, 18.1; H, 3.47; N, 5.93; Fe, 11.50; Mo, 7.18. IR (KBr, cm $\left.{ }^{-1}\right): 1$ 521(s), 1 384(s), 1 259(s), $1241(\mathrm{~m})$, 1 265(m), 1 176(sh), 1 162(s), 994(m), 948(s), 851(m), 704(m), 336(s), 315(m), 303(m), 275(w).

(2) $\left[\mathrm{MoFe}_{3} \mathrm{Se}_{4}\left(\mathrm{Et}_{2} \mathrm{dtc}\right)_{6}\right](2)$ 的制备. $\left[\mathrm{Et}_{4} \mathrm{~N}\right]_{2}\left[\mathrm{MoSe}_{4}\right](0.17 \mathrm{~g}, 0.25 \mathrm{mmol}), \mathrm{FeCl}_{2}(0.10 \mathrm{~g}, 0.79$ $\mathrm{mmol})$ 和 $\mathrm{Et}_{2} \mathrm{dtcNa}(0.26 \mathrm{~g}, 1.50 \mathrm{mmol})$ 溶于 $20 \mathrm{~mL} \mathrm{DMF} / \mathrm{CH}_{3} \mathrm{CN}$ (体积比为 $1: 1$ )中, 室温下 搅拌过夜. 保持滤液于 $4^{\circ} \mathrm{C}$, 几周后, 可得到用于 $\mathrm{X}$ 射线分析的黑色棱柱状晶体, 用无水乙 醇洗涤并真空干燥得 $0.12 \mathrm{~g}$ (产率为 $32.3 \%$ ). 按 $\mathrm{C}_{30} \mathrm{H}_{60} \mathrm{~N}_{6} \mathrm{MoFe}_{3} \mathrm{Se}_{4} \mathrm{~S}_{12}$ 计算值 $(\%)$ : C, 24.5; H, 4.12; N, 5.72; S, 26.2; Fe, 11.4; Mo, 6.53. 实验值(\%): C, 25.1; H, 4.41; N, 5.81; S, 25.8; Fe, 10.9; Mo, 6.38. IR (KBr, cm $\left.{ }^{-1}\right): 1$ 487(s), 1 441(vs), 1 391(s), 1 331(s), 1 244(m), 1 271(m), 1 181(sh), 1 170(s), 1 106(w), 1 007(m), 948(m), 913(w), 833(m), 703(m), 335(s), 318(m), 303(m), 280(w).

(3) $\left[\mathrm{MoFe}_{3} \mathrm{Se}_{4}\left(\mathrm{C}_{4} \mathrm{H}_{8} \mathrm{dtc}\right)_{6}\right](3)$ 的制备. 同 1 的方法, 只是用 $\mathrm{C}_{4} \mathrm{H}_{8} \mathrm{dtcNa}(1.27 \mathrm{~g}, 1.60 \mathrm{mmol})$ 来代替 $\mathrm{Me}_{2} \mathrm{dtcNa}$ 盐. 保持滤液于 $-10^{\circ} \mathrm{C}$ 下, 几天后, 分离出黑色针状晶体 $0.11 \mathrm{~g}$ (产率为 $30.6 \%$ ). 按 $\mathrm{C}_{30} \mathrm{H}_{48} \mathrm{~N}_{6} \mathrm{MoFe}_{3} \mathrm{Se}_{4} \mathrm{~S}_{12}$ 计算值 (\%): C, 24.7; H, 3.32; N, 5.77; Fe, 11.5; Mo, 6.59. 实验值 (\%): $\mathrm{C}$, 25.6; H, 4.31; N, 5.66; Fe, 10.8; Mo, 7.04. IR (KBr, cm $\left.{ }^{-1}\right): 1$ 493(s), 1 401(vs), $1356(\mathrm{~m}), 1302(\mathrm{~m})$, 1 271(s), 1 209(s), 1 147(m), 1 077(s), 913(w), 779(m), 668(m), 361(m), 331(s), 311(m), 304(m), 260(w).

\section{2 晶体结构分析}

选择合适的化合物 2 的单晶用 $\mathrm{AB}$ 胶固定在玻璃丝上端, 以 Siemens Smart CCD 面探测 仪，在 $23^{\circ} \mathrm{C}$ 下用石墨化 Mo-K $\alpha$ 射线测定单胞参数和用 $\omega$ 扫描技术于 $3.5<2 \theta<50^{\circ}$ 范围内收 集数据. 数据还原在 Silicon Graphics 工作站用 Smart CCD 软件进行.

结构计算在 HP/586 微机上用 SHELXL-PC 程序包进行. 重原子坐标由直接法解出, 其余 非氢原子通过差值 Fowier 电子密度图得出, 应用 SHELXL-93 程序包通过 $\mathrm{F}^{2}$ 对结构进行修正. 所有非氢原子经各向异性修正, 氢原子的位置按 $\mathrm{C}-\mathrm{H}$ 键长为 $0.096 \mathrm{~nm}$ 固定在相关的碳原子 上进行修正. 最后一轮全矩阵最小二乘法修正包括 3284 个 $(F \geqslant 4.0 \sigma(F))$ 可观察点和 254 个可 变参数. 金属原子按统计分布处理, 最后一轮修正表明 4 个金属原子占有率基本相等.

\section{3 其他物理测试}

红外光谱在 FTS-40 光谱仪上用 $\mathrm{KBr}$ 压片测得. 电化学循环伏安图在装有 $X-Y$ 记录仪的 DHZ-1 多功能电化学仪器上测定. 用 SCE 为参比电极, 铂网为工作电极, Pt 丝为辅助电极, 以 ${ }^{n} \mathrm{Bu}_{4} \mathrm{NBF}_{4}(0.1 \mathrm{~mol} / \mathrm{L})$ 为支持电解质, 扫描速度为 $100 \mathrm{mV} / \mathrm{s}$, 样品溶在 $\mathrm{DMSO}$ 中, 浓度范围在 $0.6 \times 10^{-3} \sim 1.5 \times 10^{-3} \mathrm{~mol} / \mathrm{L}$.

\section{2 结果与讨论}

\section{1 合成与反应体系}

类似于含硫立方烷簇合物, 含 $\mathrm{MoFe}_{3} \mathrm{Se}_{4}$ 单立方烷核芯簇合物 $\left[\mathrm{MoFe}_{3} \mathrm{Se}_{4}\left(\mathrm{R}_{2} \mathrm{NCS}_{2}\right)_{6}\right]\left(\mathrm{R}_{2}=\right.$ $\mathrm{Me}_{2}, \mathrm{Et}_{2}, \mathrm{C}_{4} \mathrm{H}_{8}$ 很容易由一步反应制得:

$$
\left[\mathrm{MoSe}_{4}\right]^{2-}+3 \mathrm{FeCl}_{2}+6 \mathrm{R}_{2} \mathrm{dtc}^{-} \longrightarrow\left[\mathrm{MoFe}_{3} \mathrm{Se}_{4}\left(\mathrm{R}_{2} \mathrm{dtc}\right)_{6}\right]+6 \mathrm{Cl}^{-}
$$

值得注意的是, 尽管用 $\mathrm{Fe}^{2+}$ 离子为起始原料, 但是在产物中有高于 $\mathrm{Fe}^{2+}$ 离子, 很明显, 在反应 
过程中存在由低价态 $\mathrm{Fe}^{2+}$ 向高价态铁离子转变的不均衡反应. $\mathrm{R}_{2} \mathrm{NCS}_{2}$-配体显示两种配位方式: 即螯合 1 个金属原子和桥联 2 个金属原子以使得每个金属原子满足六配位的环境, 正是这种 饱和的配位几何使得最后的产物很稳定. 在我们的反应体系中, $\mathrm{R}_{2} \mathrm{NCS}_{2}{ }^{-}$为双齿配体, 因而这 些自发形成的自组装反应行为明显不同于用单齿硫配体所得到的双立方烷产物 ${ }^{[7]}$; 而且在标 题化合物中 $\mathrm{MoFe}_{3} \mathrm{Se}_{4}$ 核芯的表观氧化态至少是+6 价, 这比在 $\mathrm{Mo}_{2} \mathrm{Fe}_{6} \mathrm{Se}_{8}$ 中的每个+3 价的 $\mathrm{MoFe}_{3} \mathrm{Se}_{4}$ 核芯的氧化态要高得多 ${ }^{[7]}$. 与同类硫体系的结果一样 ${ }^{[10,}{ }^{11]}$, 标题化合物中簇核的高氧 化态是由于 $\mathrm{R}_{2} \mathrm{NCS}_{2}{ }^{-}$配体的特殊性所致, 其还原能力较单齿硫醇配体要低得多, 而且在溶液 中共振形式的多样性表现出多种配位方式. 共振形式及 C 的配位方式如下:

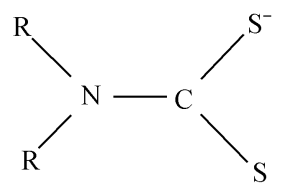

A

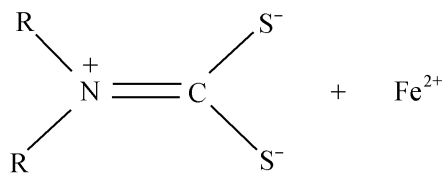

C

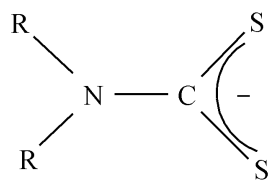

$\mathrm{B}$

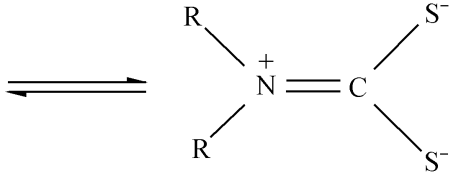

C

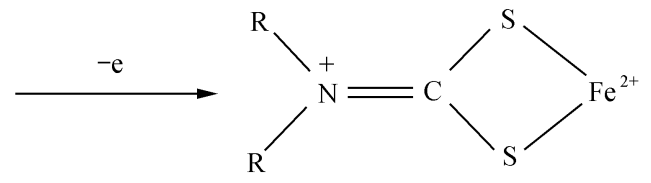

$\mathrm{D}$

构型 $\mathrm{A}$ 往往为单齿配位, 构型 $\mathrm{B}$ 和 $\mathrm{C}$ 为多齿配位, 其中 $\mathrm{B}$ 一般表现为螯合配位, 而在构 型 $\mathrm{C}$ 中富电子的 $\mathrm{S}$ 原子很容易与高氧化态的金属离子配位. 当用 $\mathrm{Fe}^{2+}$ 为起始物时, 即使在严 格绝氧的条件下, 很微量 $\mathrm{O}_{2}{ }^{[12]}$ 也能使 $\mathrm{Fe}^{2+}$ 离子失去一个电子变成高价态 $\mathrm{Fe}$ 离子, 并进而与 以构型 $\mathrm{C}$ 共振形式存在的 $\mathrm{R}_{2} \mathrm{NCS}_{2}$-配体反应形成活性碎片 $\left[\mathrm{FeS}_{2} \mathrm{CNR}_{2}\right]^{2+}$. 这与我们很难分离出 含 $\mathrm{R}_{2} \mathrm{NCS}_{2}{ }^{-}$单纯二价铁产物的事实一致. 正是因为在反应体系中具有丰富配位形式 $\mathrm{R}_{2} \mathrm{NCS}_{2}{ }^{-}$ 配体和高价态 $\mathrm{Fe}$ 离子的存在, 使得具有高氧化态簇核化合物的形式. 这些结果与电化学循环 伏安研究中观察到的 $\mathrm{MoFe}_{3} \mathrm{Se}_{4}$ 簇核可以转变到更高的价态 $(+7$ 和 +8$)$ 的结果是一致的.

\section{$2.2\left[\mathrm{MoFe}_{3} \mathrm{Se}_{4}\left(\mathrm{Et}_{2} \mathrm{dtc}\right)_{6}\right](2)$ 的结构}

$X$ 射线结构测定测得化合物 2 的晶体学数据列于表 1 , 坐标及热参数列于表 2 . 表 3 给出 化合物 2 的主要键长和键角. 测定结果表明化合物 2 的晶体结构是含有由 2 个桥式和 4 个端 基鳌合的 $\mathrm{Et}_{2} \mathrm{NCS}_{2}$-配体所包围的一个扭曲类立方烷 $\mathrm{M}_{4} \mathrm{Se}_{4}$ 簇核, 其核结构类似于 $\mathrm{MoFe}_{3} \mathrm{~S}_{4}\left(\mathrm{Me}_{2} \mathrm{NCS}_{2}\right)_{6}$. 簇合物的结构如图 1 所示, 它具有通过立方烷簇心的一对菱形平面 $\mathrm{M}(1) \mathrm{M}(1 \mathrm{a}) \mathrm{Se}(1) \mathrm{Se}(1 \mathrm{a})$ 和 $\mathrm{M}(2) \mathrm{M}(2 \mathrm{a}) \operatorname{Se}(2) \operatorname{Se}(2 \mathrm{a})$ 的两个中心点的晶体学的 $\mathrm{C}_{2}$ 轴. 化合物 2 中的 4 个金属原子具有等同的六配位几何环境, 以至于彼此之间不能区分, 使得它们在簇骼中成统 计分布, 即 $M(1)=M(2)=M(1 a)=M(2 a)=(1 / 4) \mathrm{Mo}+(3 / 4) F e$. 由 $\mathrm{Et}_{2} \mathrm{NCS}_{2}$-配体桥连的 $\mathrm{M}(1)$ 一 $\mathrm{M}(2)$ 和 $\mathrm{M}(1 \mathrm{a})-\mathrm{M}(2 \mathrm{a})$ 的键长 0.2741 (2) $\mathrm{nm}$ 明显要短于其他在 $0.2780(3)$ 和 $0.3024(2) \mathrm{nm}$ 范 围内 $\mathrm{M}-\mathrm{M}$ 键长, 化合物中所有 $\mathrm{M}-\mathrm{M}$ 键长的平均值为 $0.2834(2) \mathrm{nm}$, 比相应 Mo-Fe-S 簇合物 
表 1 化合物 2 的晶体数据和结构参数

\begin{tabular}{ll}
\hline 化合物 & $\mathrm{MoFe}_{3} \mathrm{Se}_{4}\left(\mathrm{Et}_{2} \mathrm{NCS}_{2}\right)_{6}$ \\
\hline 分子式 & $\mathrm{C}_{30} \mathrm{H}_{60} \mathrm{~N}_{6} \mathrm{MoFe}_{3} \mathrm{Se}_{4} \mathrm{~S}_{12}$ \\
分子量 & 1468.9 \\
空间群 & $\mathrm{C} 2 / \mathrm{c}$ \\
$a / \mathrm{nm}$ & $3.0356(6)$ \\
$b / \mathrm{nm}$ & $1.0592(3)$ \\
$c / \mathrm{nm}$ & $2.0571(3)$ \\
$\beta /\left(^{\circ}\right)$ & $120.98(3)$ \\
$V / \mathrm{nm} m^{3}$ & $5.671(3)$ \\
分子数 & 4 \\
计算密度 $/ \mathrm{g} \cdot \mathrm{cm}^{-3}$ & 1.772 \\
温度/K & 296 \\
$\mu / \mathrm{mm}^{-1}$ & 3.745 \\
波长 $/ \mathrm{nm}(\mathrm{Mo}-\mathrm{K} \alpha)$ & 0.071073 \\
$F(000)$ & 2987 \\
$2 \theta /\left({ }^{\circ}\right)$ & $1.45 \sim 24.48$ \\
独立衍射点数 & 4036 \\
观察衍射点数 & $3284(F \geqslant 4 \sigma(F))$ \\
变数 & 254 \\
$R\left(F_{0}\right)$ 值 & 0.061 \\
$R_{\mathrm{w}}\left(F_{0}\right)$ 值 & 0.073 \\
$S$ 值 & 2.18 \\
最高和最低残余峰 $/ \mathrm{e}(\mathrm{nm})^{-3}$ & $0.81,-0.85$ \\
\hline
\end{tabular}

表 2 化合物 2 的原子坐标及热参数

\begin{tabular}{|c|c|c|c|c|}
\hline 原子 & $x \times 10^{4} / \mathrm{nm}$ & $y \times 10^{4} / \mathrm{nm}$ & $z \times 10^{4} / \mathrm{nm}$ & $U($ eq $) \times 10^{3} / \mathrm{J}$ \\
\hline $\mathrm{M}(1)$ & $4608(1)$ & $1498(1)$ & $7660(1)$ & $30(1)$ \\
\hline $\mathrm{M}(2)$ & $4561(1)$ & $3488(1)$ & $6776(1)$ & $32(1)$ \\
\hline $\operatorname{Se}(1)$ & $4487(1)$ & $1313(1)$ & $6434(1)$ & $38(1)$ \\
\hline $\operatorname{Se}(2)$ & $4566(1)$ & $3676(1)$ & $7926(1)$ & $42(1)$ \\
\hline$S(1)$ & $3666(1)$ & $1361(3)$ & $7056(2)$ & $49(2)$ \\
\hline$S(2)$ & $3612(1)$ & $3627(3)$ & $6121(2)$ & $47(1)$ \\
\hline$S(3)$ & $4338(1)$ & $4135(2)$ & $5485(2)$ & $43(1)$ \\
\hline$S(4)$ & $4474(1)$ & $5796(2)$ & $6651(2)$ & $34(1)$ \\
\hline$S(5)$ & $4506(1)$ & $840(2)$ & $8732(2)$ & $36(1)$ \\
\hline$S(6)$ & $4523(1)$ & $-817(2)$ & $7657(2)$ & $34(1)$ \\
\hline $\mathrm{N}(1)$ & $2842(4)$ & $2666(10)$ & $6184(7)$ & $70(6)$ \\
\hline $\mathrm{N}(2)$ & $4243(3)$ & $6648(8)$ & $5286(5)$ & $48(5)$ \\
\hline $\mathrm{N}(3)$ & $4360(3)$ & $-1650(7)$ & $8745(5)$ & $34(4)$ \\
\hline$C(1)$ & $3337(4)$ & $2584(10)$ & $6434(6)$ & $42(6)$ \\
\hline $\mathrm{C}(2)$ & $2517(4)$ & $3733(14)$ & $5682(9)$ & $85(9)$ \\
\hline $\mathrm{C}(3)$ & $2557(5)$ & $4855(15)$ & $6105(11)$ & $122(13)$ \\
\hline $\mathrm{C}(4)$ & $2497(11)$ & $1338(35)$ & $6274(13)$ & $278(30)$ \\
\hline $\mathrm{C}(5)$ & $2580(8)$ & $1903(21)$ & $6827(17)$ & $232(27)$ \\
\hline$C(6)$ & $4334(3)$ & $5661(8)$ & $5750(6)$ & $32(5)$ \\
\hline $\mathrm{C}(7)$ & $4196(4)$ & $7920(9)$ & $5526(7)$ & $54(6)$ \\
\hline $\mathrm{C}(8)$ & $3716(5)$ & $8221(11)$ & $5437(9)$ & $104(10)$ \\
\hline $\mathrm{C}(9)$ & $4163(6)$ & $6435(11)$ & $4522(7)$ & $75(9)$ \\
\hline$C(10)$ & $3626(6)$ & $6472(19)$ & $3902(9)$ & $148(14)$ \\
\hline $\mathrm{C}(11)$ & $4450(3)$ & $-690(9)$ & $8404(5)$ & $31(5)$ \\
\hline$C(12)$ & $4303(4)$ & - $2937(9)$ & $8440(7)$ & $53(7)$ \\
\hline$C(13)$ & $3781(5)$ & $-3263(13)$ & $7834(10)$ & $104(11)$ \\
\hline$C(14)$ & $4299(4)$ & $-1477(9)$ & $9402(6)$ & $36(5)$ \\
\hline $\mathrm{C}(15)$ & $3746(5)$ & $-1221(13)$ & $9169(8)$ & $89(9)$ \\
\hline
\end{tabular}


表 3 化合物 2 的主要键长和键角 ${ }^{\text {a) }}$

\begin{tabular}{|c|c|c|c|}
\hline $\mathrm{M}(1)-\mathrm{M}(2)$ & $2.741(2)$ & $\mathrm{M}(2)-\mathrm{Se}(2)$ & $2.366(2)$ \\
\hline$M(1)-M(1 a)$ & $2.780(3)$ & $M(2)-\operatorname{Se}(2 a)$ & $2.401(2)$ \\
\hline $\mathrm{M}(1)-\mathrm{M}(2 \mathrm{a})$ & $3.024(2)$ & $\mathrm{M}(1)-\mathrm{S}(1)$ & $2.466(3)$ \\
\hline$M(2)-M(2 a)$ & $2.792(2)$ & $M(1)-S(5)$ & $2.478(4)$ \\
\hline $\mathrm{M}(1)-\mathrm{Se}(1)$ & $2.364(2)$ & $M(1)-S(6)$ & $2.464(3)$ \\
\hline $\mathrm{M}(1)-\mathrm{Se}(2)$ & $2.389(2)$ & $\mathrm{M}(2)-\mathrm{S}(2)$ & $2.479(3)$ \\
\hline $\mathrm{M}(1)-\mathrm{Se}(1 \mathrm{a})$ & $2.406(1)$ & $\mathrm{M}(2)-\mathrm{S}(3)$ & $2.476(3)$ \\
\hline $\mathrm{M}(2)-\mathrm{Se}(1)$ & $2.386(1)$ & $\mathrm{M}(2)-\mathrm{S}(4)$ & $2.458(3)$ \\
\hline $\mathrm{Se}(1)-\mathrm{M}(1)-\mathrm{Se}(2)$ & $109.0(1)$ & $S(1)-M(1)-S(5)$ & $77.8(1)$ \\
\hline $\mathrm{Se}(1)-\mathrm{M}(1)-\mathrm{Se}(1 \mathrm{a})$ & $107.9(1)$ & $S(1)-M(1)-S(6)$ & $81.2(1)$ \\
\hline $\mathrm{Se}(2)-\mathrm{M}(1)-\mathrm{Se}(1 \mathrm{a})$ & $94.3(1)$ & $S(5)-M(1)-S(6)$ & $70.3(1)$ \\
\hline $\mathrm{Se}(1)-\mathrm{M}(2)-\mathrm{Se}(2)$ & 109.1(1) & $\mathrm{S}(2)-\mathrm{M}(2)-\mathrm{S}(3)$ & $78.5(1)$ \\
\hline $\mathrm{Se}(1)-\mathrm{M}(2)-\mathrm{Se}(2 \mathrm{a})$ & $94.5(1)$ & $S(2)-M(2)-S(4)$ & $81.5(1)$ \\
\hline $\mathrm{Se}(2)-\mathrm{M}(2)-\mathrm{Se}(2 \mathrm{a})$ & $107.5(1)$ & $\mathrm{S}(3)-\mathrm{M}(2)-\mathrm{S}(4)$ & $69.9(1)$ \\
\hline $\operatorname{Se}(1)-\mathrm{M}(1)-\mathrm{S}(1)$ & $87.4(1)$ & $\mathrm{S}(5)-\mathrm{M}(1)-\mathrm{Se}(1 \mathrm{a})$ & $84.5(1)$ \\
\hline $\mathrm{Se}(2)-\mathrm{M}(1)-\mathrm{S}(1)$ & $89.3(1)$ & $\mathrm{S}(1)-\mathrm{M}(1)-\mathrm{Se}(1 \mathrm{a})$ & $162.0(1)$ \\
\hline $\mathrm{Se}(1)-\mathrm{M}(1)-\mathrm{S}(5)$ & $154.8(1)$ & $\mathrm{S}(6)-\mathrm{M}(1)-\mathrm{Se}(1 \mathrm{a})$ & $89.9(1)$ \\
\hline $\mathrm{Se}(2)-\mathrm{M}(1)-\mathrm{S}(5)$ & $91.3(1)$ & $\mathrm{S}(2)-\mathrm{M}(2)-\mathrm{Se}(2 \mathrm{a})$ & $162.8(1)$ \\
\hline $\mathrm{Se}(1)-\mathrm{M}(1)-\mathrm{S}(6)$ & $87.5(1)$ & $\mathrm{S}(3)-\mathrm{M}(2)-\mathrm{Se}(2 \mathrm{a})$ & $84.7(1)$ \\
\hline $\mathrm{Se}(2)-\mathrm{M}(1)-\mathrm{S}(6)$ & $160.6(1)$ & $\mathrm{S}(4)-\mathrm{M}(2)-\mathrm{Se}(2 \mathrm{a})$ & $89.4(1)$ \\
\hline $\mathrm{Se}(1)-\mathrm{M}(2)-\mathrm{S}(2)$ & $89.4(1)$ & $\mathrm{M}(1)-\mathrm{Se}(1)-\mathrm{M}(2)$ & $70.5(1)$ \\
\hline $\mathrm{Se}(2)-\mathrm{M}(2)-\mathrm{S}(2)$ & $86.9(1)$ & $\mathrm{M}(1)-\mathrm{Se}(1)-\mathrm{M}(1 \mathrm{a})$ & 71.3(1) \\
\hline $\mathrm{Se}(1)-\mathrm{M}(2)-\mathrm{S}(3)$ & $91.1(1)$ & $\mathrm{M}(2)-\mathrm{Se}(1)-\mathrm{M}(1 \mathrm{a})$ & $78.3(1)$ \\
\hline $\mathrm{Se}(2)-\mathrm{M}(2)-\mathrm{S}(3)$ & $155.0(1)$ & $\mathrm{M}(1)-\mathrm{Se}(2)-\mathrm{M}(2)$ & $70.4(1)$ \\
\hline $\mathrm{Se}(1)-\mathrm{M}(2)-\mathrm{S}(4)$ & $160.2(1)$ & $\mathrm{M}(1)-\mathrm{Se}(2)-\mathrm{M}(2 \mathrm{a})$ & $78.3(1)$ \\
\hline $\mathrm{Se}(2)-\mathrm{M}(2)-\mathrm{S}(4)$ & $88.1(1)$ & $\mathrm{M}(2)-\mathrm{Se}(2)-\mathrm{M}(2 \mathrm{a})$ & $71.7(1)$ \\
\hline
\end{tabular}

a) 键长单位为 $\mathrm{nm}$, 键角单位为 $\left(^{\circ}\right)$

中的要略长, 如在 $\mathrm{MoFe}_{3} \mathrm{~S}_{4}\left(\mathrm{Me}_{2} \mathrm{NCS}_{2}\right)_{6}$ 中为 $0.2801(3) \mathrm{nm}^{[10]}$, 在 $\mathrm{MoFe}_{3} \mathrm{~S}_{4}\left(\mathrm{Me}_{2} \mathrm{NCS}_{2}\right)_{5}$ 中为 0.278 1(4) $\mathrm{nm}^{[11]}$; 所有 $\mathrm{M}$-Se 键长在 0.236 4(2)到 $0.2406(1) \mathrm{nm}$ 的范围内, 平均值为 $0.2389(2) \mathrm{nm}$, 比 相应 $\mathrm{M}-\mathrm{S}(\mathrm{M}=\mathrm{Mo}, \mathrm{Fe})$ 的键长要长, 这是由于硒比硫有较大的原子半径所致. 然而, 在 2 中 平均 $\mathrm{M}$ - $\mathrm{Se}$ 键长比在 $\left\{\mathrm{MoAg}_{3} \mathrm{Se}{ }_{3} \mathrm{Cl}\right\}\left(\mathrm{PPh}_{3}\right)_{3} \mathrm{Se}(0.2360(3) \mathrm{nm})^{[13]}$ 和 $\left\{\mathrm{MoCu}_{3} \mathrm{Se}_{3} \mathrm{Cl}\right\}\left(\mathrm{PPh}_{3}\right)_{3} \mathrm{Se}$ $(0.2370(4) \mathrm{nm})^{[9]}$ 中的 $\mathrm{Mo}-\mathrm{Se}_{\mathrm{b}}$ 要略长, 但比在 $\left\{\mathrm{MoAg}_{3} \mathrm{Se}_{3} \mathrm{Cl}\right\}\left(\mathrm{PPh}_{3}\right)_{3} \mathrm{Se}$ 中的 $\mathrm{Ag}-\mathrm{Se}(0.2621(2)$ $\mathrm{nm})$ 的键长 ${ }^{[13]}$ 和在 $\left\{\mathrm{MoCu}_{3} \mathrm{Se}_{3} \mathrm{Cl}\right\}\left(\mathrm{PPh}_{3}\right)_{3} \mathrm{Se}^{[9]}$ 中 $\mathrm{Cu}-\mathrm{Se}(0.2409(6) \mathrm{nm})$ 的键长要明显的短, 其原 因可以归结为在 $\mathrm{Mo}-\mathrm{Fe}-\mathrm{Se}$ 簇核中金属原子的平均分布及其所形成的协同效应, 这一点 Holm 在 $\mathrm{Fe}-\mathrm{Se}$ 簇合物中已有论证 ${ }^{[14]} . \mathrm{M}-\mathrm{S}_{\mathrm{dtc}}\left(\mathrm{dtc}=\mathrm{Et}_{2} \mathrm{NCS}_{2}{ }^{-}\right)$键长变化范围在 $0.2458(3) \sim 0.2479(3)$ $\mathrm{nm}$ 之间, 与同时在含 $\mathrm{R}_{2} \mathrm{NCS}_{2}{ }^{-}(\mathrm{R}=\mathrm{Et}, \mathrm{Me}$ )配体 Mo-Fe-S 立方烷(键长范围为 $0.2413(4) \sim$

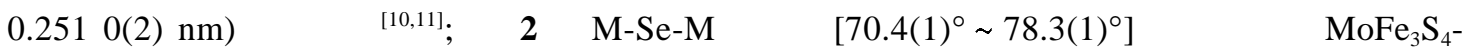
$\left(\mathrm{Me}_{2} \mathrm{NCS}_{2}\right)_{6}$ 中相应的键角 $\left[75.7(1)^{\circ} \sim 81.4(1)^{\circ}\right]^{[15]}$.

\section{3 循环伏安行为}

表 4 给出簇合物 $\left[\mathrm{MoFe}_{3} \mathrm{Se}_{4}\left(\mathrm{R}_{2} \mathrm{dtc}\right)_{6}\right]\left(\mathrm{R}_{2}=\mathrm{Me}_{2}, \mathrm{Et}_{2}, \mathrm{C}_{4} \mathrm{H}_{8}\right)$ 的氧化还原电位, 其循环伏安谱如 图 2 所示. 在 $+0.5 \sim-2.0 \mathrm{~V}$ 扫描范围内, 可以观察到 3 个可分辨的氧化还原峰, 半波电位 $E_{1 / 2}$ 分别在 $+0.10 \sim 0.13 \mathrm{~V},-0.20 \sim-0.28 \mathrm{~V}$ 和 $-0.14 \sim-0.19 \mathrm{~V}$ 之间. 刘秋田等人 ${ }^{[11]}$ 曾报道

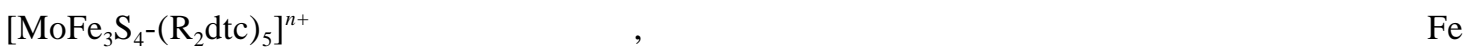
原子之间氧化还原转化所致, 而六价 Mo(VI)的电子轨道并没有特别的贡献. 这一结论在 


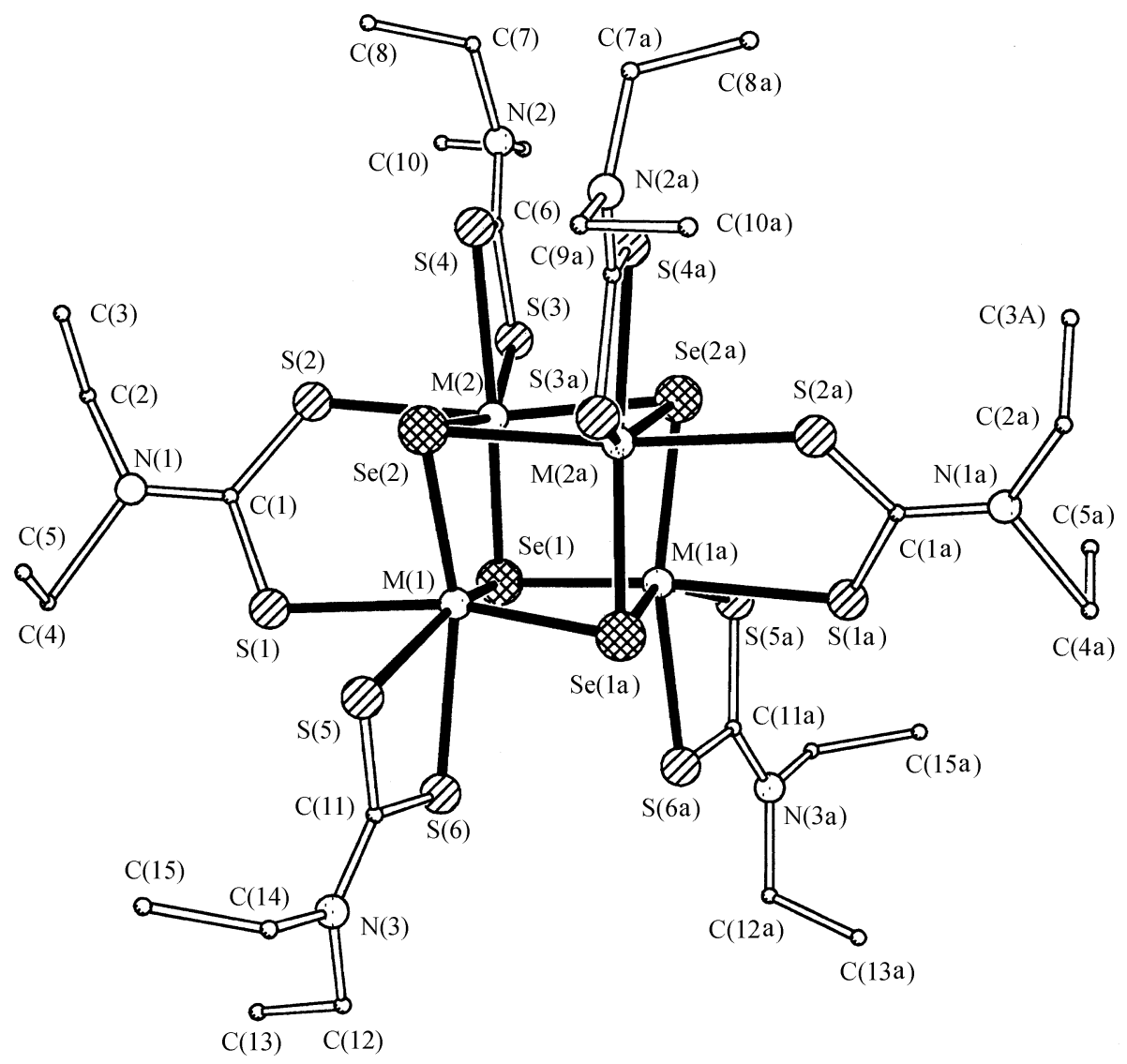

图 $1 \mathrm{MoFe}_{3} \mathrm{Se}_{4}\left(\mu-\mathrm{Et}_{2} \mathrm{NCS}_{2}\right)_{2}\left(\mathrm{Et}_{2} \mathrm{NCS}_{2}\right)_{4}$ 的分子结构

我们这里得到类似的证明，亦即不同价态的 $\left[\mathrm{MoFe}_{3} \mathrm{Se}_{4}\right]$ 核芯的单电子氧化还原过程引起了可 逆的氧化还原电对，其过程可表示为:

$$
\begin{aligned}
& {\left[\mathrm{MoFe}_{3} \mathrm{Se}_{4}\left(\mathrm{R}_{2} \mathrm{dtc}\right)_{6}\right]^{2+} \stackrel{E_{1}}{\rightleftharpoons}\left[\mathrm{MoFe}_{3} \mathrm{Se}_{4}\left(\mathrm{R}_{2} \mathrm{dtc}\right)_{6}\right]^{+}} \\
& \stackrel{E_{2}}{\rightleftharpoons}\left[\mathrm{MoFe}_{3} \mathrm{Se}_{4}\left(\mathrm{R}_{2} \mathrm{dtc}\right)_{6}\right]^{0} \stackrel{E_{3}}{\rightleftharpoons}\left[\mathrm{MoFe}_{3} \mathrm{Se}_{4}\left(\mathrm{R}_{2} \mathrm{dtc}\right)_{6}\right]^{-}
\end{aligned}
$$

在这一变化序列中, 可观察到 $\left[\mathrm{MoFe}_{3} \mathrm{Se}_{4}\right]^{8+}$, 表明在簇合物、循环伏安过程中存在 $\mathrm{Fe}^{4+} ; \mathrm{Holm}$ 等人 ${ }^{[16]}$ 曾在簇合物 $\left[\mathrm{Fe}_{6} \mathrm{~S}_{8}\left(\mathrm{PEt}_{3}\right)_{6}\right]^{n+}(n=3,4)$ 中报道过类似的结果. 这些结论表明高价态簇骼可

\begin{tabular}{|c|c|c|c|c|c|c|c|c|c|c|c|c|}
\hline \multirow{2}{*}{ 化合物 ${ }^{b)} R_{2}$} & \multicolumn{4}{|c|}{$2+/ 1+$} & \multicolumn{4}{|c|}{$1+/ 0$} & \multicolumn{4}{|c|}{$0 / 1-$} \\
\hline & $E_{\mathrm{p}, \mathrm{c}}$ & $E_{\mathrm{p}, \mathrm{a}}$ & $E_{1 / 2}$ & $\Delta E_{\mathrm{p}}$ & $\overline{E_{\mathrm{p}, \mathrm{c}}}$ & $E_{\mathrm{p}, \mathrm{a}}$ & $E_{1 / 2}$ & $\Delta E_{\mathrm{p}}$ & $\overline{E_{\mathrm{p}, \mathrm{c}}}$ & $E_{\mathrm{p}, \mathrm{a}}$ & $E_{1 / 2}$ & $\Delta E_{\mathrm{p}}$ \\
\hline $\mathrm{Et}_{2}(\mathbf{2})$ & +0.05 & +0.14 & +0.10 & 0.09 & -0.32 & -0.24 & -0.28 & 0.08 & -1.23 & -1.15 & -1.19 & 0.08 \\
\hline $\mathrm{Me}_{2}(\mathbf{1})$ & +0.08 & +0.18 & +0.13 & 0.08 & -0.24 & -0.15 & -0.20 & 0.09 & -1.20 & -1.08 & -1.14 & 0.12 \\
\hline $\mathrm{C}_{4} \mathrm{H}_{8}(3)$ & +0.07 & +0.13 & +0.10 & 0.06 & -0.28 & -0.19 & -0.24 & 0.09 & -1.21 & -1.10 & -1.15 & 0.11 \\
\hline
\end{tabular}
表 4 化合物 2 的配合物 $\left[\mathrm{MoFe}_{3} \mathrm{Se}_{4}\left(\mathrm{R}_{2} \mathrm{dtc}\right)_{6}\right]^{\mathrm{a}}$ 相对 $\mathrm{SCE}$ 岸电极的氧化还原电位/ $\mathrm{V}$

a) $\mathrm{Pt}$ 网工作电极, $\mathrm{Pt}$ 丝辅助电极, 支持电解质为 $\mathrm{n}-\mathrm{Bu}_{4} \mathrm{NBF}_{4}$, 扫描速度为 $100 \mathrm{mV} / \mathrm{s}$

b) DMSO 溶液 

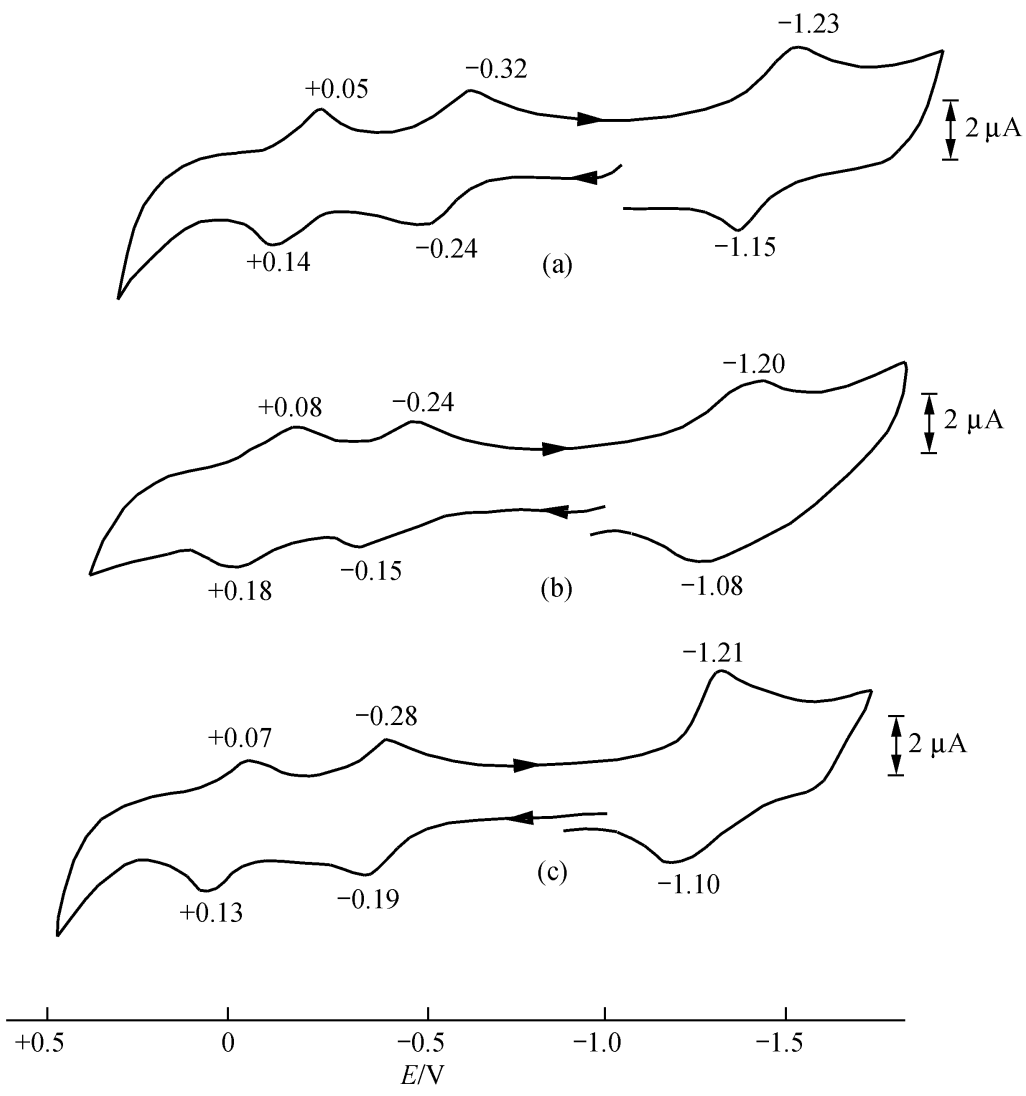

图 2 室温下 $\mathrm{MoFe}_{3} \mathrm{Se}_{4}\left(\mu-\mathrm{R}_{2} \mathrm{NCS}_{2}\right)_{2}\left(\mathrm{R}_{2} \mathrm{NCS}_{2}\right)_{4}\left(\mathrm{R}_{2}=\mathrm{Me}_{2}(\mathrm{a}), \mathrm{Et}_{2}(\mathrm{~b}), \mathrm{C}_{4} \mathrm{H}_{8}(\mathrm{c})\right)$ 的循环伏安图

$\mathrm{Pt}$ 网工作电极, $\mathrm{Pt}$ 丝辅助电极, 支持电解质为 $\mathrm{n}-\mathrm{Bu}_{4} \mathrm{NBF}_{4}$, 扫描速度为 $100 \mathrm{mv} / \mathrm{s}$

以由配位的 $\mathrm{R}_{2} \mathrm{dtc}$ 配体得到稳定, 这与刘秋田先期的报道 ${ }^{[11]}$ 完全一致. 尽管 Mo-Fe-Se 簇合物 与之具有几乎等同的结构类型, 但是, 由较长相似的结果的键参数所引起的较大的电子位移 可以导致在循环伏安实验中产生丰富的氧化还原变化.

\section{参 考 文 献}

1 Coucouvanis D. Fe-M-S complexes derived from $\mathrm{MS}_{4}{ }^{2-}$ anions $(\mathrm{M}=\mathrm{Mo}, \mathrm{W})$ and their possible as analogues for structural features in the Mo site of nitrogenase. Acc Chem Res, 1981, 14: $210 \sim 219$

2 Armstrong W H, Mascharak P K, Holm R H. Demostration of the existence of single cubance-type $\mathrm{MoFe}_{3} \mathrm{~S}_{4}$ clusters with S = 3/2 ground states: prepation, structure, and properties. Inorg Chem, 1982, 21: $1699 \sim 1701$

3 Kim J, Rees D C. Crystallographic structure and functional implications of the nitrogenance molybdenum-iron protein from azotobacter vinelandii. Nature, 1992, 360: $553 \sim 560$

4 Demadis K D, Campana C F, Coucouvanis D. Synthesis and structural characterization of the new $\mathrm{Mo}_{2} \mathrm{Fe}_{6} \mathrm{~S}_{8}\left(\mathrm{PR}_{3}\right)_{6}\left(\mathrm{Cl}_{4}-\mathrm{cat}_{2}\right.$ cluster. Doble cubanes containing two edge-linked $\left[\mathrm{MoFeS}_{4}\right]^{2+}$ reduced cores. J Am Chem Soc, 1995, 117: $7832 \sim 7833$ Stadtman T C. Selenium protein in nature. Science, 1974, 183: $915 \sim 921$

6 Sola M, Cowan J A, Gray H B. ${ }^{1} \mathrm{H}$ NMR spectra and electron-transfer properties of oxidized and reduced $\left[\mathrm{Fe}_{4} \mathrm{~S}_{4}\right]$ derivatives 
of clotridium vinosum high-potential iron protein. J Am Chem Soc, 1989, 111: $6627 \sim 6630$

7 Greaney M A, Coyle C L, Pilato R S, et al. Synthesis and characterization of "double cubane" complexes containing $\mathrm{MFe}_{3} \mathrm{~S}_{4}$ cores $(\mathrm{M}=\mathrm{Mo}, \mathrm{W})$. Inorg Chim Acta, 1991, 189: $81 \sim 96$

8 O' Neal S C, Kolis J W. Convenient preparation and structures of selenometalates $\mathrm{MoSe}_{4}{ }^{2-}, \mathrm{WSe}_{4}{ }^{2-}$ and $\mathrm{MoSe}_{9}{ }^{2-}$ from polyselenide anion and metal carbonyls. J Am Chem Soc, 1988, 110: $1971 \sim 1973$

9 Zhang Q F, Hong M C, Su W P, et al. Syntheses and spectroscopic properties of complexes with the cubane-like core $\left\{\mathrm{MoCu}_{3} \mathrm{Se}_{3} \mathrm{X}\right\}(\mathrm{X}=\mathrm{Cl}, \mathrm{Br}, \mathrm{I})$. Crystal structure of $\left\{\mathrm{MoCu}_{3} \mathrm{Se}_{3} \mathrm{Cl}\right\}\left(\mathrm{PPh}_{3}\right)_{3} \mathrm{Se}$. Polyhedron, 1997, 16: $1433 \sim 1437$

10 刘秋田, 黄梁仁, 杨 瑜, 等. $\mathrm{MoFe}_{3} \mathrm{~S}_{4}$ 单立方烷原子簇研究. IV. 新型双桥单立方烷簇合物 $\mathrm{MoFe}_{3} \mathrm{~S}_{4}\left(\mathrm{MeNCS}_{2}\right)_{6} \cdot \mathrm{CH}_{3} \mathrm{CN}$ 的合 成和结构. 化学学报, 1988, 46: $1075 \sim 1081$

11 Liu Q T, Huang L R, Liu H Q, et al. Structural chemistry of molybdenum-iron-sulfur cluster compounds with a single cubane $\left[\mathrm{MoFe}_{3} \mathrm{~S}_{4}\right]^{n+}(n=4 \sim 6)$ core and crystal structure of $\left[\mathrm{MoFe}_{3} \mathrm{~S}_{4}\left(\mathrm{MeNCS}_{2}\right)_{5}\right] \mathrm{CH}_{2} \mathrm{Cl}_{2}$. Inorg Chem, 1990, $29: 4131 \sim 4137$

12 Kawaguchi H, Yamada K, Lang J P, et al. A new entry into molybdenum/tungsten sulfur chemistry: syntheses and reactions of mononuclear sulfido complexes of pentamethylcyclopentadieyl- molybdenum (VI) and-tungsten. J Am Chem Soc, 1997, 119: $10346 \sim 10358$

13 Zhang Q F, Hong M C, Liu H Q. Syntheses of the Mo-Ag-Se complexes with a cubane-like core $\left\{\mathrm{MoAg}_{3} \mathrm{Se}_{3} \mathrm{X}\right\}(\mathrm{X}=\mathrm{Cl}, \mathrm{Br})$. Crystal structure of $\left\{\mathrm{MoAg}_{3} \mathrm{Se}_{3} \mathrm{Cl}\right\}\left(\mathrm{PPh}_{3}\right)_{3} \mathrm{Se}$. Trans Met Chem, 1997, 22: $156 \sim 169$

14 Yu S B, Papaefthymiou G C, Holm R H. Comprehensive iron-selenium-thiolate cluster chemistry. Inorg Chem, 1991, 30: $3476 \sim 3485$

15 刘秋田, 雷新建, 黄梁仁, 等. $\mathrm{MoFe}_{3} \mathrm{~S}_{4}$ 及 $\mathrm{WFe}_{3} \mathrm{~S}_{4}$ 单立方烷簇合物的合成、结构和电化学研究. 中国科学, B 辑, 1990, (5): $561 \sim 563$

16 Goddard C A, Long J R, Holm R H. Synthesis and characterization of four consecutive members of the five-member $\left[\mathrm{Fe}_{6} \mathrm{~S}_{8}\left(\mathrm{PEt}_{3}\right)_{6}\right]^{n+}(n=0 \sim 4)$. Inorg Chem, 1996, 35: $4347 \sim 4354$ 